\title{
Contributions of Supercoiling to Tn3 Resolvase and Phage Mu Gin Site-specific Recombination
}

\author{
Kirsten R. Benjamin, A. Pia Abola, Roland Kanaar and \\ Nicholas R. Cozzarelli*
}

Division of Biochemistry and Molecular Biology

Department of Molecular and Cell Biology, 401 Barker Hall University of California Berkeley, CA 94720-3204

USA

\begin{abstract}
Members of the resolvase/invertase family of site-specific recombinases require supercoiled substrates containing two recombination sites. To dissect the roles of supercoiling in recombination by the Tn 3 and $\gamma \delta$ resolvases and the phage $\mathrm{Mu}$ Gin invertase, we used substrates that provided some but not all of the topological features of the standard substrate. We divided the Tn3 resolvase reaction into two stages, synapsis and postsynapsis. We found the contributions of supercoiling to each stage were distinct, since substrate catenation in the absence of supercoiling or low levels of substrate supercoiling were sufficient for synapsis but not postsynapsis. Using structural and functional topological analyses, we verified that the resolvase synaptic complexes with nicked catenanes were recombination intermediates. The requirement for supercoiling was even less stringent for the $\gamma \delta$ resolvase, which recombined nicked catenanes about half as well as it did supercoiled substrates. Gin recombination of catenanes occurred even if the recombinational enhancer was on a nicked ring, as long as both crossover sites were on a supercoiled ring. Therefore, supercoiling is required at the Gin crossover sites but not at the enhancer. We conclude that solely conformational effects of supercoiling are required for resolvase synapsis and the function of the Gin enhancer, but that a torsional effect, probably double helix unwinding, is needed for Tn3 resolvase postsynapsis and at the Gin recombination sites.

(C) 1996 Academic Press Limited
\end{abstract}

Keywords: site-specific recombination; supercoiling; resolvase; Gin; catenanes work, we address the roles of supercoiling in site-specific recombination catalyzed by three members of the resolvase/invertase family (Hatfull \& Grindley, 1988; Feng et al., 1994). We focus on the Tn3 resolvase and briefly investigate the $\gamma \delta$ resolvase and the phage $\mathrm{Mu}$ Gin invertase.

The resolvases are needed for the second reaction in replicative transposition of the $\operatorname{Tn} 3$ and $\gamma \delta$ transposons. They catalyze the conversion of the circular cointegrate intermediate into two catenated product circles by recombining sequences internal to the transposons (reviewed by Hatfull \& Grindley, 1988; Sherratt, 1989; Grindley, 1994; Stark \& Boocock, 1995). Gin catalysis results in the alternate expression of two sets of phage $\mathrm{Mu}$ tail fiber genes by inverting a DNA segment in the phage genome (reviewed by Hatfull \& Grindley, 1988; Glasgow et al., 1989; Johnson, 1991). Invertase and resolvase recombination depend on supercoiling in vitro (Reed, 1981; Krasnow \& Cozzarelli, 1983; Mertens et al., 1984; Plasterk et al., 1984; Johnson \& Simon, 
1985); a parallel dependence in vivo has been demonstrated for Tn3 resolvase (Bliska et al., 1991).

The resolvase and invertase reactions are shown in Figure 1. For both classes of enzyme, the standard substrate is a supercoiled circular DNA with two recombination sites that bind the recombinase. For resolvase recombination, the res sites must be directly repeated. For Gin recombination, the gix sites must be inversely repeated and an accessory site called the recombinational enhancer (sis) is also required to bind the host factor FIS. The standard reaction mechanism is as follows (Figure 1). After resolvase or Gin plus FIS bind their DNA sites, the sites come together to form a complex in a process called synapsis. The sites are interwound in both synaptic complexes, trapping three DNA crossings or nodes with resolvase (Wasserman et al., 1985; Benjamin \& Cozzarelli, 1988) and two with Gin and FIS (Kanaar et al., 1988; Heichman \& Johnson, 1990). Resolvase synapsis can be directly assayed by lowering the reaction temperature from $37^{\circ} \mathrm{C}$ to $15^{\circ} \mathrm{C}$; at the lower temperature, synapsis is rapid and efficient, but later steps are very slow (Benjamin \& Cozzarelli, 1988). The second stage of the reaction, called postsynapsis or strand exchange, consists of recombinase-catalyzed double-stranded breakage of both crossover sites, rearrangement, and religation of the DNA in the recombinant configuration. Dissociation of the complexes reveals a singly-linked catenane for resolvase recombination and a circle containing an inverted segment for Gin recombination (Figure 1). Whereas the product of Gin recombination can undergo subsequent rounds of recombination, that of resolvase cannot, due to topological restrictions (Krasnow \& Cozzarelli, 1983; Stark et al., 1989).

Some of the roles of (-) supercoiling in recombination have been demonstrated (reviewed by Kanaar \& Cozzarelli, 1992). Supercoiling favors synapsis by providing the nodes that interwind the sites in the synaptic complex (Craigie \& Mizuuchi, 1986; Kanaar \& Cozzarelli, 1992; Stark \& Boocock, 1995). The second known effect of supercoiling is on strand exchange. In (-) supercoiled DNA, strand exchange always occurs by a right-handed rotation of the cleaved half sites to give a unique product with a lower superhelical density than the substrate (Kanaar et al., 1988; Stark et al., 1989; Heichman et al., 1991; Stark \& Boocock, 1994; see also Figure 1). Under special conditions where the need for supercoiling is bypassed, recombination occurs with rotations in both directions (Stark et al., 1989; Klippel et al., 1993).

A useful tool for investigating the roles of supercoiling in recombination has been catenated DNA, in which the recombination sites are partitioned between two rings (Craigie \& Mizuuchi, 1986; Kanaar et al., 1989; Stark et al., 1989; Benjamin \& Cozzarelli, 1990). Recombination with supercoiled catenane substrates retains its dependence on the orientation of the recombination sites (Craigie \& Mizuuchi, 1986; Kanaar et al., 1989; Benjamin and Cozzarelli, 1990; Adams et al., 1992).
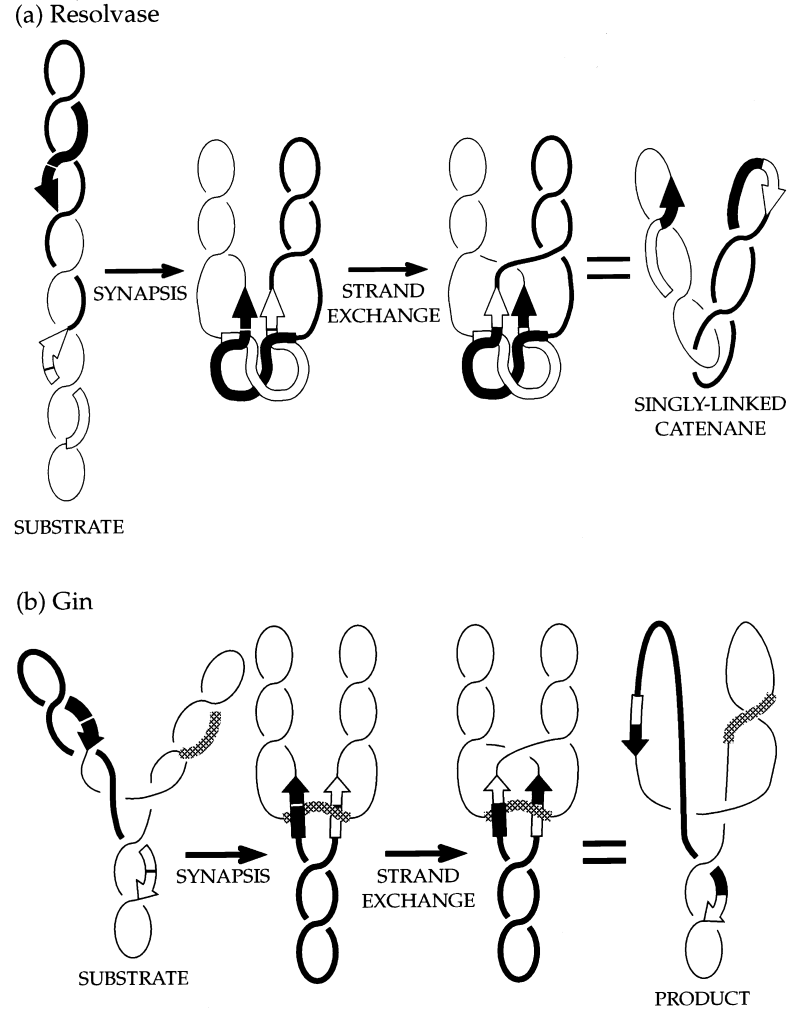

Figure 1. Resolvase and Gin recombination. Recombination is divided into two steps: synapsis (juxtaposition of recombination sites) and postsynapsis or strand exchange (breakage and crossed reunion of sites). Synapsis interwinds the res sites with three (-) superhelical crossings and the Gin sites with two (-) superhelical crossings. During postsynapsis, the recombinase cleaves the DNA at the centers of the crossover sites, rearranges the broken sites by a $180^{\circ}$ rotation, and religates them in the recombinant configuration. We define a right-handed rotation as one that increases the twist of the DNA by +1 and therefore removes (-) supercoils. Double-stranded DNA is represented by a single line and therefore alterations of twist are not shown. (a) Resolvase recombines a supercoiled substrate containing two directly repeated, $120 \mathrm{bp}$ recombination sites, res. The res sites bound by resolvase are depicted by arrows. Each res is composed of three subsites, I, II, and III, each of which binds a resolvase dimer; subsite I is the crossover site. The product is a singly-linked (two-noded) catenane. (b) Gin recombines a supercoiled substrate containing two inversely repeated, $34 \mathrm{bp}$ recombination crossover sites, gix, and one accessory site, the recombinational enhancer sis. The Gin-bound gix sites are depicted by arrows and the speckled box represents the FIS-bound sis. Gin synapsis interwinds the three sites at a branch point in the substrate. Since the DNA double helix is represented by a single line, the diagrams do not accurately reflect the change in linking number that occurs during resolvase and Gin recombination.

Catenation mimics the conformational but not the torsional effects of supercoiling (Vologodskii \& Cozzarelli, 1993). Conformational effects include the arrangement of the DNA axis in three-dimensional space, particularly the interactions between segments on the same molecule (reviewed by 
Vologodskii \& Cozzarelli, 1994). Torsional effects include the distortion of the double helix, for example, by unwinding or melting.

Catenation can provide some, but not all, of the contributions of supercoiling to recombination. Resolvase and Gin can recombine two separate supercoiled rings (each of which contains one recombination site) when they are catenated by multiple interlinks, but not when the rings are unlinked or singly-linked (Krasnow \& Cozzarelli, 1983; Kanaar et al., 1989; Stark et al., 1989; Benjamin \& Cozzarelli, 1990). Because the recombination sites are on separate rings, catenane nodes must substitute for supercoil nodes in the synaptic complex, yet the multiply-linked catenanes still require supercoiling for recombination. This suggests that the conformational features shared by supercoiling and catenation are necessary for recombination, but are not sufficient on their own.

We address three questions in this work. (1) Is supercoiling required for resolvase synapsis, postsynapsis, or both stages? (2) Is the contribution of supercoiling to each stage the same? (3) Is supercoiling of one DNA ring in the catenane sufficient for recombination? We used two approaches to answer these questions. First, we measured the dependence of Tn 3 resolvase synapsis and recombination on the superhelical density of circular substrates. Second, we used catenated substrates to dissect the roles of supercoiling in resolvase and Gin recombination. We found that supercoiling at low levels or catenation alone promotes proper $\mathrm{Tn} 3$ resolvase synapsis but not steps after synapsis, demonstrating that the need for supercoiling is more stringent for postsynapsis than synapsis. Also, we observed recombination with some catenanes that had only one supercoiled ring, showing that supercoiling is dispensable for one resolvase recombination site, res, and for the Gin recombinational enhancer, sis. We interpret these results with reference to the structural properties of catenanes and supercoiled circles. We conclude that the critical mechanistic assistance provided by supercoiling to synapsis and the accessory site depends on the conformational effects, and that the boost to postsynapsis and the crossover sites relies on torsional stress.

\section{Results}

\section{Recombination requires a higher superhelical density than synapsis}

We first determined whether Tn3 resolvase synapsis and recombination respond differently to superhelical density, $\sigma$. Synapsis is much faster than recombination: under standard conditions, synapsis is complete before $200 \mathrm{~ms}$ and recombination is mostly complete after ten minutes (Parker \& Halford, 1991; Oram et al., 1994). We generally measure synapsis by an agarose gel mobility shift assay after brief incubation of resolvase with its substrate at $15^{\circ} \mathrm{C}$ to minimize recombination,

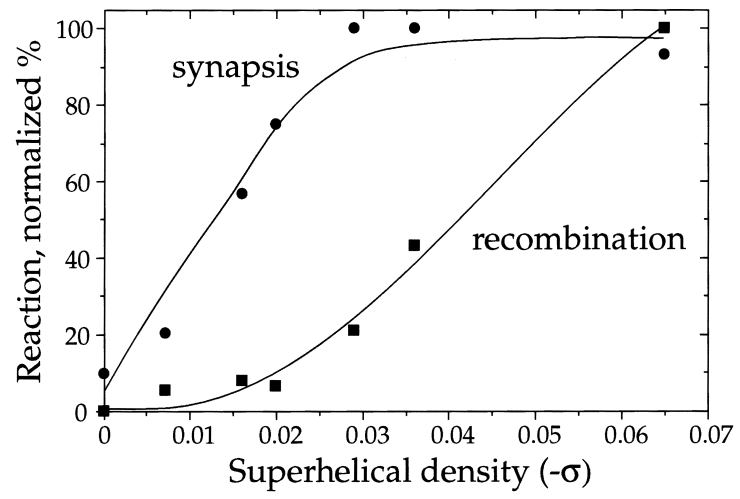

Figure 2. Effect of superhelical density on synapsis and recombination. The dependencies on substrate superhelical density $(\sigma)$ of the amount of synapsis ( $)$ and recombination ( $\boldsymbol{\square})$ by $\operatorname{Tn} 3$ resolvase are graphed. pResInt substrates with different average superhelical densities were assayed for synapsis or recombination after one or 60 minutes at $37^{\circ} \mathrm{C}$, respectively. To allow direct comparison of synapsis and recombination, the fraction of DNA that reacted was normalized to the highest value obtained ( $44 \%$ for synapsis and $76 \%$ for recombination).

glutaraldehyde crosslinking, and digestion of the DNA with a restriction enzyme. The synaptic complex fragment has a characteristic mobility shift because it contains two segments of DNA held together by protein. We usually measure recombination by the incubation of resolvase or Gin plus FIS with the substrate at $37^{\circ} \mathrm{C}$, digestion of the DNA with a restriction enzyme, and electrophoresis on an agarose gel. Fragments from reacted and unreacted DNA can be visualized by ethidium bromide staining or autoradiography of gels (see Materials and Methods).

In Figure 2, we used circular DNA that ranged from $\sigma=0$ (relaxed) to $\sigma=-0.065$ (superhelical

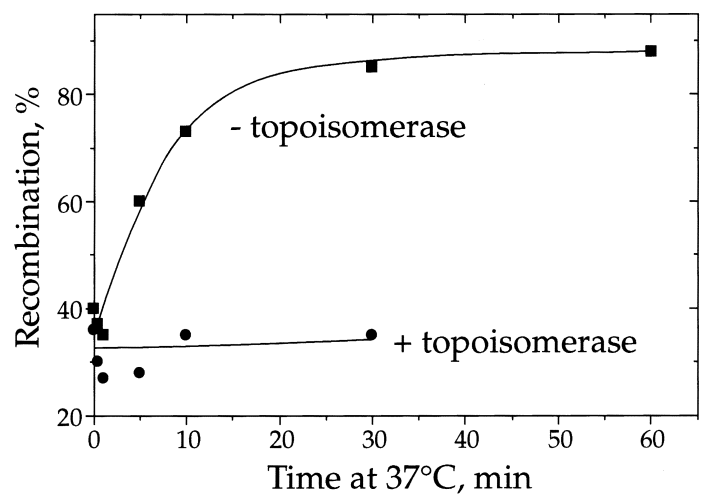

Figure 3. Recombination by $\operatorname{Tn} 3$ resolvase with a relaxed synaptic complex. Tn3 resolvase synaptic complexes formed at $15^{\circ} \mathrm{C}$ on supercoiled pResInt were treated either with wheat germ topoisomerase I or dilution buffer. The mixture was then transferred to $37^{\circ} \mathrm{C}$ at time 0 to facilitate recombination. At the indicated times, samples were assayed for recombination of the

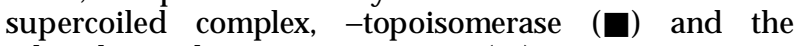
relaxed complex, +topoisomerase $(\boldsymbol{O})$. 
density of plasmids isolated from bacteria). We present the dependence on $\sigma$ of the amount of Tn3 resolvase synapsis after one minute and recombination after 60 minutes. At these time points, all the reactions had reached completion. To compare synapsis with recombination more easily, each fraction of reacted DNA was normalized to the maximum observed value. Recombination required a substantially greater level of supercoiling than did synapsis either at $37^{\circ} \mathrm{C}$ (Figure 2) or at $15^{\circ} \mathrm{C}$ (data not shown). The amount of synapsis increased linearly with increasing superhelical density, reaching a plateau of about $\sigma=-0.03$. The low level of synapsis seen with relaxed DNA was due to the topoisomer distribution around 0 that guaranteed a significant fraction of molecules with low levels of $(-)$ supercoiling. There was no synapsis with a nicked circular substrate (Benjamin \& Cozzarelli, 1988; see also Figure 6). In contrast to the results for synapsis, recombination was undetectable with relaxed DNA and remained below $8 \%$ of the maximum value until the superhelical density was greater than -0.02 . Thereafter, recombination increased steadily up to $\sigma=-0.065$. Half-maximal synapsis and recombination were observed at superhelical densities near -0.015 and -0.04 , respectively (Figure 2). We conclude that supercoiling is needed for synapsis and postsynapsis, but that higher levels are required for postsynapsis.

\section{A relaxed synaptic complex does not recombine}

We determined directly that supercoiling has a postsynaptic role by showing that relaxation of a preformed synaptic complex blocked recombination. Tn 3 resolvase synaptic complexes formed at $15^{\circ} \mathrm{C}$ on supercoiled DNA were treated with excess wheat germ topoisomerase $\mathrm{I}$, then allowed to recombine for various times at $37^{\circ} \mathrm{C}$ (Figure 3). Some recombination occurred at $15^{\circ} \mathrm{C}$ before upshift, but at $37^{\circ} \mathrm{C}$ the relaxed complex (+topoisomerase) did not recombine further, whereas the supercoiled control (-topoisomerase) reached $90 \%$ recombination. We demonstrated by the mobility shift assay that at least $75 \%$ of the synaptic complexes survived relaxation (data not shown). Hence, supercoiling of the DNA outside the synaptic complex is necessary for postsynapsis.

\section{Tn 3 resolvase can recombine hemi-nicked but not fully-nicked catenanes}

Because catenation shares some of the features of supercoiling (Vologodskii \& Cozzarelli, 1993), we used catenated substrates to understand the roles of supercoiling in site-specific recombination. We used a plasmid, pResInt, that is a substrate for both resolvase and $\lambda$ integrase (Int). Figure 4 illustrates that recombination of the supercoiled circular plasmid (called $\mathrm{S}$, for substrate) by resolvase produces singly-linked catenanes $(\mathrm{R})$; recombination by Int generates multiply-linked catenanes (I)

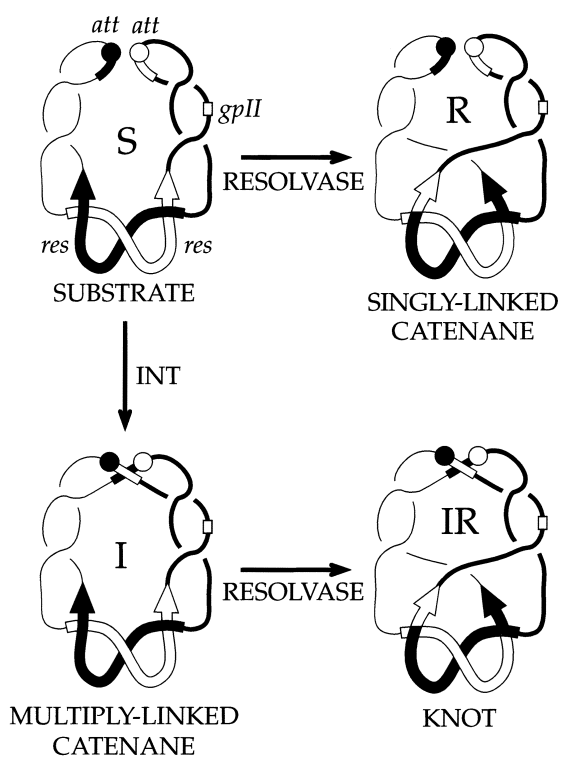

Figure 4. Catenated resolvase substrates. Derivatives of pResInt were used for resolvase reactions. This plasmid contains a gpII nicking site (box) and directly repeated $\lambda$ Int sites, att (balls and sticks), interspersed with directly repeated res sites (arrows). Resolvase recombines pResInt, called $S$ for substrate, to produce a singly-linked catenane (R) with 3.0 and $4.3 \mathrm{~kb}$ rings. Int recombination generates a mixture of multiply-linked catenanes (I) containing 3.5 and $3.8 \mathrm{~kb}$ rings; an eight-noded catenane is shown. The Int products (I) are recombined by resolvase to produce knots (IR). For clarity, supercoiling is not shown for the multiply-linked catenanes or knots. The Int catenanes can be nicked in both rings with DNase I to give fully-nicked catenanes, or specifically in one ring with the site-specific nuclease gpII to give hemi-nicked catenanes. After large scale preparative reactions and before resolvase reactions, the DNA was purified away from the preparative enzymes.

with one res on each ring; recombination by Int followed by resolvase gives knots (IR). To allow selective nicking of one catenane ring, the substrate also contains a site for a site-specific nuclease that introduces a nick, phage fd gp II (Meyer \& Geider, 1979). Both rings of the catenane could be nicked by treatment with DNase I. Thus, catenated substrates are further distinguished on the basis of their supercoiling in both, one (hemi-nicked), or neither (fully-nicked) of the rings. We use the term nicked catenanes to mean hemi-nicked and fully-nicked.

We determined whether supercoiling of one ring of a catenane was sufficient for Tn 3 resolvase recombination. Hemi-nicked catenanes might differ in reactivity from fully-nicked catenanes because the conformational free energy of the substrate is higher or because the torsional assistance of supercoiling is needed only at one site (and can perhaps be transmitted by protein-protein contacts to the other site). Large-scale preparations of supercoiled $(\sigma \approx-0.065)$, hemi-nicked, and fullynicked catenane substrates were as described above (Figure 4). The Int catenation reactions were generally $50-70 \%$ complete (Figure 5(a), lanes 1 and 
(a) Supercoiled
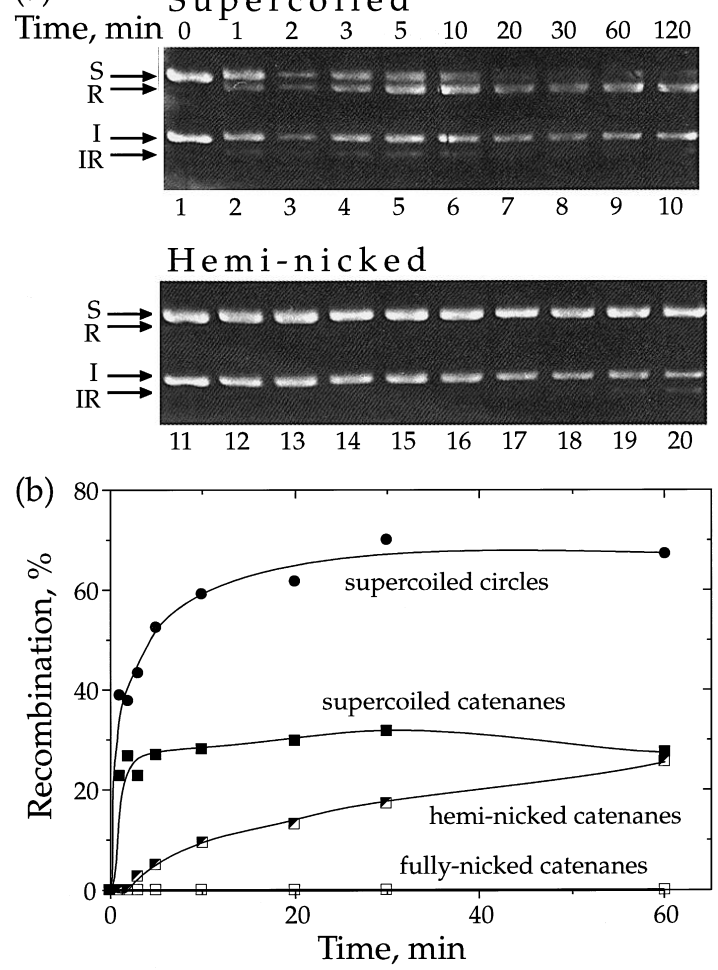

Figure 5. Tn 3 resolvase recombination with heminicked catenanes. Supercoiled and nicked catenane substrates prepared as described in Figure 4 were incubated with $\operatorname{Tn} 3$ resolvase. Recombination was assayed at the indicated time points. Samples were digested with restriction enzymes, separated by agarose gel electrophoresis, stained with ethidium bromide, photographed, and quantitated. (a) Agarose gel showing restriction fragments diagnostic of recombination with supercoiled or hemi-nicked substrates. The resolvase substrates were the catenane products of the Int reaction (I) and the residual unreacted circles (S). Recombinant bands are designated IR and R, respectively. (b) Graph of recombination of supercoiled circles $(-)$ and catenanes $(\boldsymbol{\square})$, hemi-nicked catenanes $(\boldsymbol{\nabla})$, and fully-nicked catenanes ( $\square$ ). Values from two experiments were averaged to give the results shown; each experiment alone gave a qualitatively similar result.

11) and the nicking reactions were $99 \%$ complete. After separation from the preparative enzymes, portions of the catenanes were mixed with resolvase.

A time course of $\operatorname{Tn} 3$ resolvase recombination with the three catenane substrates is shown in Figure 5(a). Resolvase clearly recombined heminicked catenanes $(I \rightarrow I R)$, but more slowly than it did supercoiled DNA $(\mathrm{S} \rightarrow \mathrm{R})$. As previously observed (Benjamin \& Cozzarelli, 1990), resolvase failed to recombine fully-nicked catenanes. The residual circular DNA unreacted by Int provided an internal control for resolvase activity and for nicking. With hemi-nicked DNA, the failure to see resolvase recombinants (R) from the circular molecules (S) demonstrated complete nicking by gpII. The data from Figure 5(a) combined with that from another experiment are shown graphically in Figure 5(b). During a 60 minute incubation, hemi-nicked catenanes were recombined to a level one third that of supercoiled circles. Therefore, although the rate and extent of recombination of hemi-nicked catenanes were lower than those of supercoiled circles, supercoiling of one res supported substantial recombination.

The apparently reduced amount of recombination with supercoiled catenanes compared to supercoiled circles (Figure 5(b)) does not accurately reflect their reactivity. Rather, recombination of supercoiled catenanes produced knots that were further recombined to form catenanes with the same restriction fragment pattern as the parental catenanes (Benjamin \& Cozzarelli, 1990). Therefore, recombination here was underestimated. Supercoiled circles are limited to one round of recombination, because the singly-linked catenane produced is not a substrate for recombination (Krasnow \& Cozzarelli, 1983; Stark et al., 1989). Detection of recombination by high-resolution gel electrophoresis (similar to Figure 8) showed that supercoiled catenanes recombined at least as well as supercoiled circles (data not shown). Recombination of nicked catenanes was limited to one round because the resultant nicked knots were not substrates of Tn 3 resolvase. Hence, the IR fragments accurately reflected recombination of nicked catenanes. Therefore, supercoiling of only one res is necessary and sufficient for Tn 3 recombination.

\section{Tn3 resolvase synapses hemi-nicked and fully-nicked catenanes}

The impaired recombination of nicked catenanes could result from an effect on synapsis or postsynapsis or both. Synapsis is easily assayed by the mobility shift assay described above. When both loops of DNA outside the synaptic complex are cleaved with a restriction enzyme, the resultant structure resembles the Greek letter $\chi$. Tn3 resolvase synapsis with fully-nicked catenanes (Figure 6(a)) or hemi-nicked catenanes (Figure 6(b)) was compared to synapsis with supercoiled catenanes. Supercoiled DNA gave a $\chi$ doublet from the synapsis of catenanes $(\chi \mathrm{I})$ and residual circles $(\chi s)$. Surprisingly, the fully-nicked catenanes yielded substantial amounts of the higher-mobility $\chi$ band $(\chi \mathrm{I})$, reflecting efficient synapsis of the fully-nicked catenanes (Figure 6(a)). In three experiments, the average amount of synapsis with fully-nicked catenanes was $60 \%$ that with supercoiled catenanes or circles. The amounts of synapsis with heminicked and supercoiled catenanes (Figure 6(b)) were essentially identical in five separate experiments. The nicked circular DNA failed to synapse (no $\chi \mathrm{s}$ for nicked substrates), as shown previously (Benjamin \& Cozzarelli, 1988). For all substrates, synapsis was complete before $15 \mathrm{~s}$.

Table 1 summarizes the Tn 3 resolvase catenane results presented thus far. Lack of supercoiling in 

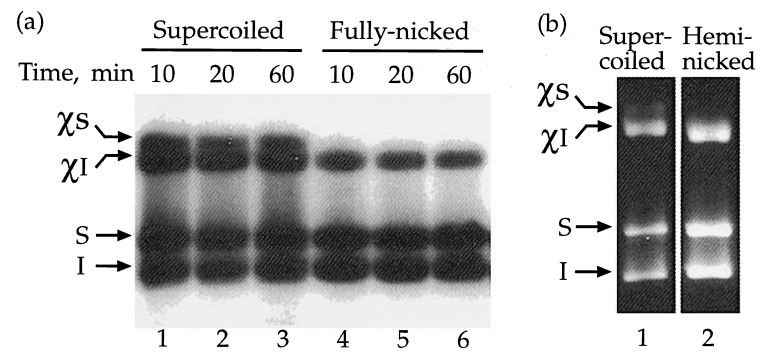

Figure 6. Tn 3 resolvase synapsis with nicked catenanes. Substrates were assayed for synapsis by $\operatorname{Tn} 3$ resolvase at $15^{\circ} \mathrm{C}$. Samples were crosslinked with glutaraldehyde, recovered by gel filtration, digested with restriction enzymes to convert the synaptic complex into a $\chi$ structure, and resolved by agarose gel electrophoresis. Bands can be visualized either by ethidium bromide staining or by autoradiography. The lower band $(\chi \mathrm{I})$ of the $\chi$ doublet in the supercoiled lanes arises from synapsis of catenanes and contains $5.5 \mathrm{~kb}$ of DNA; the upper band $(\chi s)$ is from synapsis of circular DNA left unreacted by Int and contains $6.1 \mathrm{~kb}$ of DNA. The bands indicated by I and $S$ are unsynapsed catenated and circular DNA, respectively. (a) Autoradiogram showing restriction enzyme fragments diagnostic of synapsis of supercoiled and fully-nicked catenanes after 10, 20, and 60 minutes. (b) Ethidium bromide stained gel showing synapsis of supercoiled and hemi-nicked catenanes after 15 minutes.

one or both rings of a catenane did not significantly impair synapsis. Therefore, the role of supercoiling in synapsis can be replaced by catenation. However, when supercoiling was removed from both rings of the catenane substrate, recombination was abolished. Supercoiling in one of the two rings supported significant, but reduced, levels of recombination. Therefore, the postsynaptic role of supercoiling cannot be replaced by catenation, but can be provided by just one supercoiled res. This confirms our conclusion from the superhelical density experiments that the postsynaptic requirement for supercoiling is the more stringent one.

\section{Nicked and supercoiled catenane synaptic complexes have similar topologies}

The observation that fully-nicked catenanes synapsed, but did not recombine, raised the possibility that these synaptic complexes were different from the productive ones formed with supercoiled substrates. We designed an experiment

Table 1. Tn3 resolvase reactions with circular and catenated substrates

\begin{tabular}{lcc}
\hline Substrate & Synapsis & Recombination \\
\hline Supercoiled circles & +++ & +++ \\
Nicked circles & - & - \\
Supercoiled catenanes & +++ & +++ \\
Hemi-nicked catenanes & +++ & + \\
Fully-nicked catenanes & ++ & - \\
\hline
\end{tabular}

The extents of synapsis and recombination are indicated. +++ indicates normal levels; ++ , levels $2 / 3$ of normal; and + , levels $1 / 3$ of normal. to probe the topological features of the synaptic complexes formed with catenanes. The resolvase synaptic complex on a supercoiled circle contains three superhelical crossings that interwind the two res sites (Wasserman et al., 1985; Benjamin \& Cozzarelli, 1988). On catenated substrates in which each ring contains a res, catenane nodes are the only ones that can interwind the res sites. We measured the number of catenane nodes protected from unlinking by the phage T2 type-2 topoisomerase by the formation of the synaptic complex, as shown in Figure $7(\mathrm{a})$. With a true synaptic complex, three $(-)$ nodes inside the synaptic complex should resist unlinking and one additional catenane node will remain outside the complex, because catenanes such as these must have an even number of nodes. If the outside node is $(-)$, then the catenane has a total of four nodes; alternatively, the topoisomerase can convert the outside node to $(+)$ to give a catenane with two net nodes. Thus, if the synaptic complex on nicked catenanes is like the productive one, a mixture of two and four-noded catenanes will result.

We first showed by the mobility shift assay that the synaptic complex did not decay during topoisomerase treatment. The supercoiled and nicked catenanes synapsed 25 to $50 \%$ initially and showed little change after topoisomerase treatment (Figure 7(b)). Two amounts of topoisomerase were used to show that unlinking was complete. Portions of the topoisomerase reactions were deproteinized, nicked with DNase I, and electrophoresed on a high-resolution gel. Figure 7(c) shows an autoradiogram of the gel with five lanes each for supercoiled, hemi-nicked, and fully-nicked catenanes. The first lane is the substrate alone, then a pair of lanes showing topoisomerase reactions on naked and synapsed DNA, and a second pair of lanes with double the amount of topoisomerase. The minus resolvase controls show that both amounts of topoisomerase completely decatenated the naked supercoiled and hemi-nicked substrates, and left only $4 \%$ of the fully-nicked catenanes unlinked. When the same decatenation reactions were performed on synapsed DNA, large amounts two of and four-noded catenanes were generated, as predicted by the entrapment of three $(-)$ catenane nodes in the synaptic complex. Phosphorimager quantitation revealed that approximately the same fraction of DNA accumulated as two and fournoded catenanes for all three substrates. Due to a predominance of two over four-noded catenanes, the average numbers of nodes protected by the synaptic complex was 2.4 for supercoiled catenanes and 2.2 for fully-nicked catenanes. Several variants of this experiment all gave the same result: Escherichia coli topoisomerase IV was substituted for T2 topoisomerase and $\gamma \delta$ resolvase, or its catalytic mutant S10C (Hatfull \& Grindley, 1986), was substituted for $\operatorname{Tn} 3$ resolvase (data not shown).

Therefore, the synaptic complexes with supercoiled and nicked catenanes are topologically indistinguishable. It has previously been shown that 
(a)
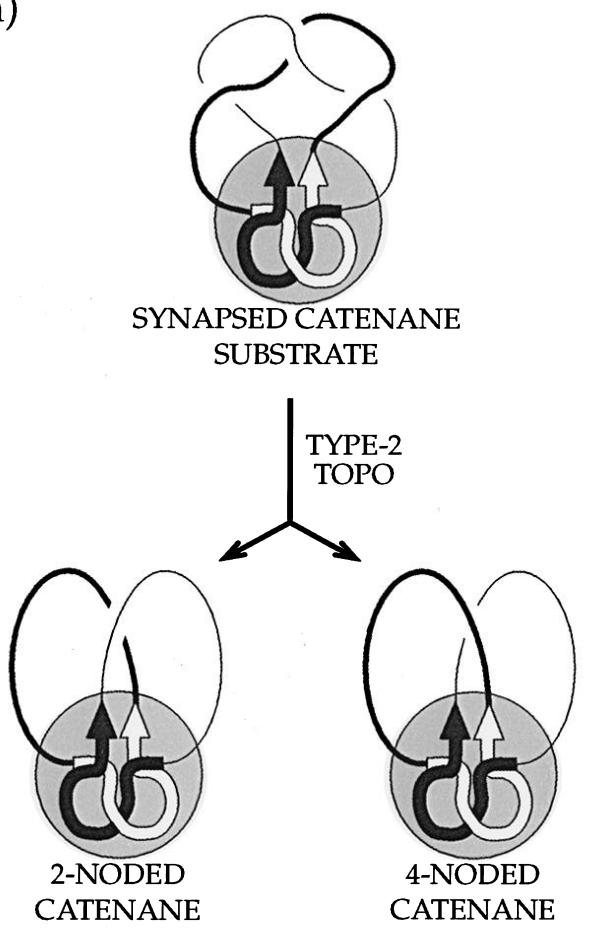

(c)
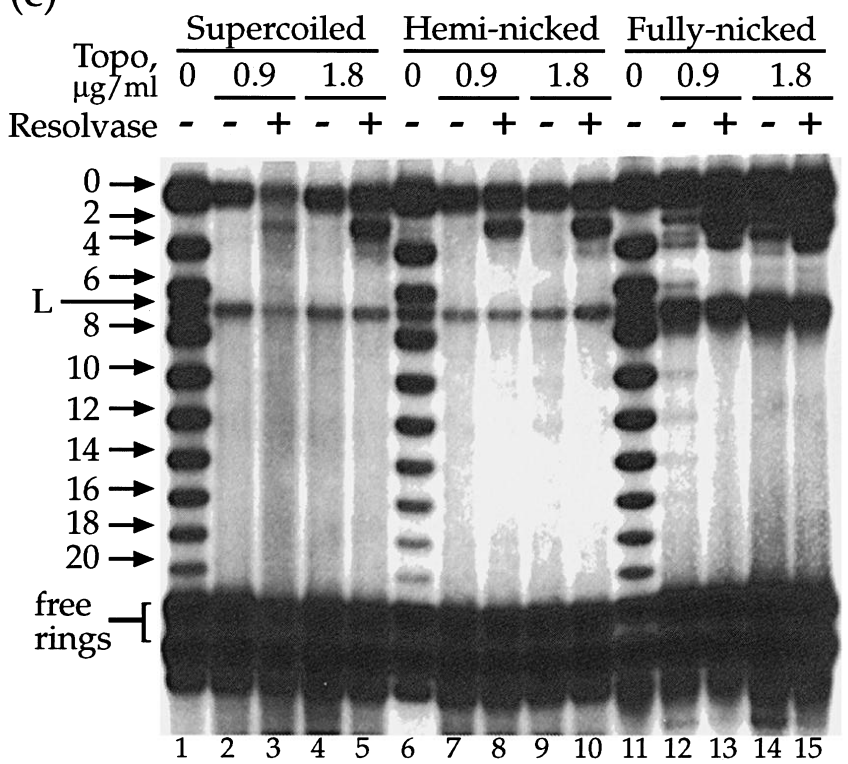

(b)

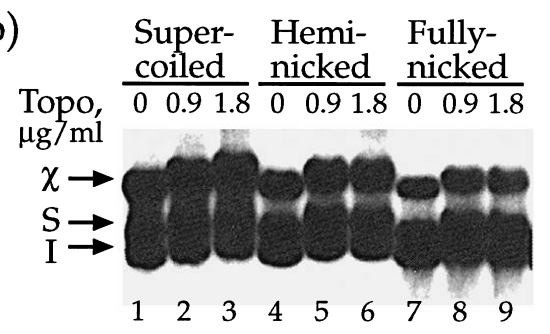

Figure 7. Protection by synapsis from type-2 topoisomerase unlinking. Supercoiled, hemi-nicked, and fully-nicked catenanes were synapsed with Tn 3 resolvase, crosslinked, and reacted with the type-2 topoisomerase from phage T2. (a) Diagram showing the expected results of treating a catenated synaptic complex with a type-2 topoisomerase. The res sites are depicted as arrows and the nucleoprotein complex as a shaded circle. Assuming three nodes are protected by the synaptic complex, reaction with topoisomerase and deproteinization will produce a mixture of two- and four-noded catenanes. (b) Autoradiogram showing restriction enzyme fragments diagnostic of Tn3 resolvase synapsis before and after topoisomerase treatment. $\chi, S$, and I are as described in Figure 6 . Two different amounts of topoisomerase were used. (c) Autoradiogram of a high resolution agarose gel showing catenane topoisomers. After topoisomerase treatment, the DNA was deproteinized, nicked with DNase I, and electrophoresed. Parallel reactions on naked (-resolvase) and synapsed (+resolvase) DNA are shown alongside the starting material. The number of nodes in each species is shown to the left of the gel. Other bands are designated L for linear parental and "free rings" for decatenated rings.

the topology of the synaptic complex with supercoiled catenanes is identical to that with supercoiled circles (Benjamin \& Cozzarelli, 1990). Thus, we conclude that the synaptic complex with each of the three catenanes, supercoiled, heminicked, and fully-nicked, is topologically identical to that with supercoiled circles.

\section{Tn3 resolvase recombines fully-nicked catenanes by the standard mechanism under special conditions}

We next performed a functional test for whether the synaptic complexes with fully-nicked and supercoiled catenanes are topologically identical.
The topology of a synaptic complex can be deduced from that of the recombination products (Wasserman \& Cozzarelli, 1986; Sumners et al., 1995). The requirement for supercoiling in recombination by Tn 3 resolvase can be partially circumvented by using reaction conditions including spermidine (Dröge \& Cozzarelli, 1989; Stark et al., 1989). Using these special conditions (Dröge \& Cozzarelli, 1989) with fully-nicked catenanes, we determined the topology of the recombination products and deduced the topology of the synaptic complex.

With a supercoiled substrate, strand exchange is mediated by a right-handed rotation of the broken sites that diminishes the number of supercoils (Figure 1(a)). Similarly, there should be a bias in the 
(a) SUBSTRATE

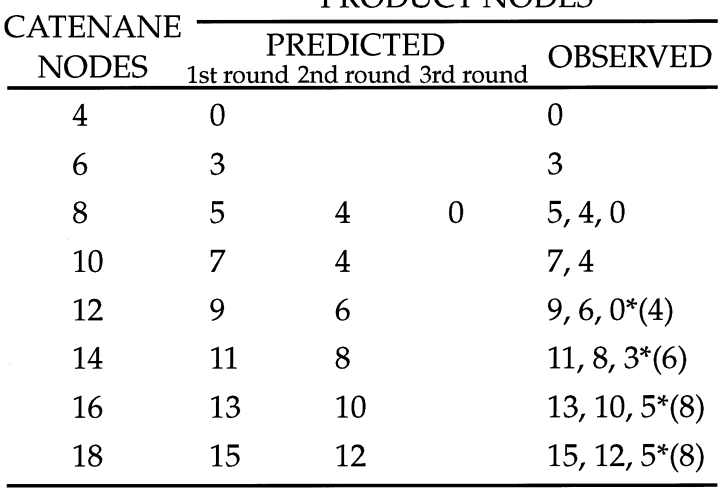

(b) Substrate $\frac{4}{\text { Resolvase }} \frac{6}{-++} \frac{8}{-++} \frac{10}{-+} \frac{12}{-+} \frac{14}{-+} \frac{16}{-++} \frac{18}{-+} \frac{\text { MIX }}{-+}$

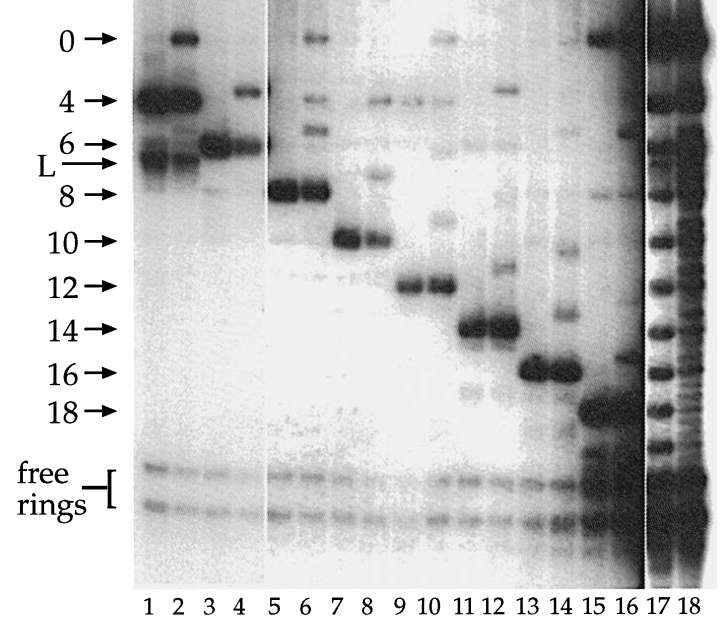

Figure 8. Tn 3 resolvase recombination of fully-nicked catenanes under permissive conditions. After reaction with Int and DNase I, fully-nicked catenane products with 4 to 18 nodes were purified according to their number of catenane nodes. Portions of the isolated species were incubated with or without Tn3 resolvase under permissive conditions for 17 hours and separated by high-resolution gel electrophoresis. (a) The predicted and observed resolvase recombination products with catenated substrates are listed according to their number of catenane and knot nodes. Predictions assumed the standard mechanism with a three-noded synaptic complex and a right-handed rotation of DNA during exchange. Asterisks denote those species that are observed but not predicted; the numbers in parentheses indicate those species, visible at low levels in the substrate lanes, from which the unexpected bands probably arise. (b) Autoradiograph showing substrates (-resolvase) and recombination products (+resolvase). Each pair of lanes is designated by the number of nodes in the predominant catenane substrate. The pair labeled "MIX" contains the mixture of catenanes produced by reaction with Int and DNase I. The identities of catenane bands are indicated to the left of the gel, as in Figure 7. Due to poor DNA recovery, lanes 5 to 16 are from an autoradiographic exposure that was four times longer than the other lanes.

direction of rotation with nicked substrates that minimizes the number of nodes in the product (Dröge \& Cozzarelli, 1989; Dröge, 1992). With catenanes, this is also a right-handed rotation.
Assuming the standard mechanism (Figure 1(a)), we deduced the products of recombination with catenanes. Figure 8(a) lists these products by their number of nodes, which is the basis of separation by electrophoresis (Benjamin \& Cozzarelli, 1990). For those catenane isomers capable of multiple rounds of recombination, the products alternate between knots and catenanes.

Fully-nicked catenane topoisomer substrates ranging in complexity from four to 18 nodes were recombined using Tn3 resolvase (Figure 8(b)). Substrates were prepared by reaction with Int and DNase I to produce a mixture of catenanes (MIX) which were then purified by high-resolution gel electrophoresis. The isolated substrates contained more than $85 \%$ of the predominant isomer, except the four-noded preparation, which was only $60 \%$ pure. Under standard recombination conditions, Tn 3 resolvase failed to recombine even the most complex (18-noded) nicked catenanes (data not shown). However, with permissive recombination conditions and overnight incubation, recombination of the nicked catenanes was substantial (16 to $46 \%$; Figure $8(\mathrm{~b})$ ). Each catenane isomer gave the species predicted from the standard mechanism of recombination. This is most easily seen by comparison of the "predicted" and "observed" columns in Figure 8(a).

The few minor bands in the 12 to 18 noded catenane lanes that were not predicted are probably products of contaminating catenane isomers. These products are listed with asterisks in Figure 8(a) followed by their parental isomer in parentheses. For example, the open circular DNA seen after recombination of the 12-noded sample is the predicted product of the contaminating four-noded catenane substrate. We ruled out the possibility that the unexpected species arose from a left-handed rotation during exchange. A catenane with $n$ nodes will produce a knot with $n+1$ nodes after a left-handed rotation, yet the unexpected species all have fewer nodes than the substrate.

The results demonstrate that under permissive conditions, fully-nicked catenanes formed the genuine synaptic complex and were recombined by the standard mechanism. Only those products resulting from right-handed strand rotation were observed; thus, we conclude that catenation dictates the direction of rotation. This experiment substantiates the conclusion that the synaptic complex formed on nicked catenanes under standard conditions is genuine, and therefore that supercoiling has specific postsynaptic role(s).

\section{$\gamma \delta$ resolvase, in contrast to $\operatorname{Tn} 3$ resolvase, recombines nicked catenanes under standard conditions}

The $\operatorname{Tn} 3$ and $\gamma \delta$ resolvases are functionally interchangeable in standard reactions and $80 \%$ identical in amino acid sequence (Hatfull \& Grindley, 1988; Sherratt, 1989). It is usually assumed that they are essentially equivalent (but see Stark 


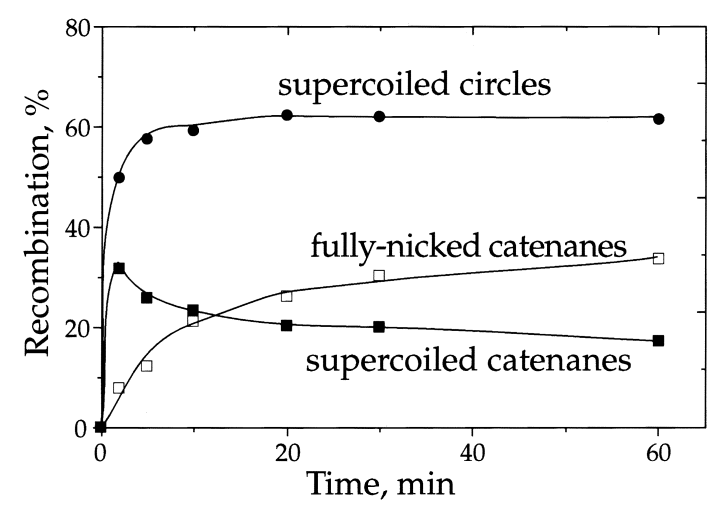

Figure 9. $\gamma \delta$ resolvase recombination with fully-nicked catenanes. $\gamma \delta$ resolvase recombination of supercoiled circles $(\mathbf{)})$ and catenanes $(\boldsymbol{\square})$ and fully-nicked catenanes ( $\square$ ). The procedure was as described in Figure 5.

et al., 1991). However, the literature contains apparently conflicting results on resolvase recombination of nicked knots (Dröge \& Cozzarelli, 1989; Dröge, 1992). The first study found that resolvase recombined nicked knots only under the special conditions used above (Dröge \& Cozzarelli, 1989). Later, it was observed that resolvase recombined nicked knots under standard conditions (Dröge, 1992). Although the conflict was not addressed, one difference between the two papers was the use of $\operatorname{Tn} 3$ or $\gamma \delta$ resolvase. The results can be reconciled if it is assumed that $\gamma \delta$ resolvase has a much less stringent dependence on supercoiling than Tn3 resolvase.

We tested this hypothesis with our fully-nicked catenane substrate. Both enzymes recombined supercoiled circles at almost the same rate and nicked circles not at all (Figures 5 and 9).

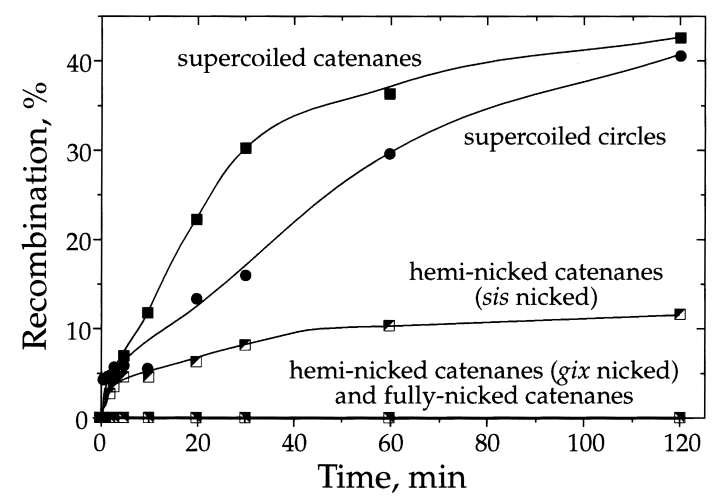

Figure 10. Gin recombination with hemi-nicked catenanes. Graph of Gin recombination of catenanes over time. Recombination was assayed as described for resolvase. Hemi-nicked (sis) indicates catenanes that contained sis on a nicked ring $(\boldsymbol{\nabla})$; hemi-nicked (gix) contained a gix on a nicked ring $(\boldsymbol{\nabla})$. The data for both fully-nicked catenanes are also represented by $\boldsymbol{\nabla}$. Data for supercoiled catenanes $(\boldsymbol{\square})$ and circles $(\boldsymbol{O})$ are shown for the plasmid that can be converted into hemi-nicked (sis); similar results were seen for the other Gin substrate. The data show the average of two experiments; each experiment alone gave similar results.
However, $\gamma \delta$ resolvase recombined the fullynicked catenanes to a level $30 \%$ that of the supercoiled circles after ten minutes (Figure 9), whereas no reaction was observed with $\operatorname{Tn} 3$ resolvase (Figure 5(b)). During a 60 minute incubation, $\gamma \delta$ resolvase recombined fully-nicked catenanes to a level half that of supercoiled circles. The initial rate is about tenfold faster with supercoiled catenanes than fully-nicked catenanes. Because the resolvase substrate used throughout this study contains $\gamma \delta$ res sites, it was possible that $\gamma \delta$ resolvase differed from Tn 3 resolvase because of a pairing of $\gamma \delta$ sites with Tn3 enzyme. However, we found that the difference between the resolvases was unaffected by whether the substrate contained $\gamma \delta$ or Tn 3 res sites (data not shown). We conclude that the postsynaptic requirement for supercoiling is much less stringent for $\gamma \delta$ than $\operatorname{Tn} 3$ resolvase and that the postsynaptic role of supercoiling is not obligatory for the reaction even under standard conditions.

\section{Gin recombination with hemi-nicked catenanes requires supercoiling of both gix sites but not of sis}

We obtained further insight into the roles of supercoiling in recombination by using heminicked catenane substrates for the Gin invertase. The Gin system is related in many ways to the resolvase system (Hatfull \& Grindley, 1988; Stark \& Boocock, 1995). A major difference between Gin and resolvase lies in the sites required for recombination. A res site is a hybrid of a crossover site (subsite I), where the cleavage occurs, and accessory sites (subsites II and III) that are critical for synapsis and activation of catalysis (Grindley, 1994; Stark \& Boocock, 1995). The Gin crossover site gix is analogous to subsite I, and the accessory site sis may be functionally equivalent to res subsites II and III (Johnson, 1991; Stark \& Boocock, 1995). Therefore, Gin recombination of hemi-nicked catenanes offered an opportunity to dissect the DNA sites into crossover and accessory site functions and to determine whether supercoiling is required for one or both functions.

The two hemi-nicked catenane substrates we used differed in whether the ring nicked by gpII contains sis or gix. A time course of Gin recombination is shown in Figure 10. We confirmed that Gin recombined supercoiled catenanes even better than the supercoiled circular substrate and failed to recombine fully-nicked catenanes (Kanaar et al., 1989). No recombination was detected when a gix was on the nicked ring, even when the other gix and sis were on the supercoiled ring (heminicked $(g i x))$. However, when the nicked ring contained sis and both gix were on the supercoiled ring (hemi-nicked (sis)), Gin recombination approached $30 \%$ that of supercoiled DNA.

Therefore, catenation can replace some of the roles of supercoiling in Tn3 resolvase and Gin reactions. Catenation alone substitutes for super- 
coiling in resolvase synapsis and Gin accessory site function. However, catenation cannot support Tn3 resolvase postsynapsis or Gin recombination site activity. We discuss below how we think supercoiling is contributing to each aspect of these reactions.

\section{Discussion}

We have analyzed the requirement for (-) supercoiling in the Tn 3 and $\gamma \delta$ resolvase and Gin invertase reactions. To dissect the roles of supercoiling we used substrates with nonstandard topology that provided some but not all of the features of the standard supercoiled substrate. We divide the resolvase reaction into two parts, synapsis and postsynapsis. We found that supercoiling is required for $\operatorname{Tn} 3$ resolvase synapsis and postsynapsis, and that catenation alone, or low levels of supercoiling, sufficed for the former but not the latter. Just as catenation replaced supercoiling for one element of the resolvase reaction, it replaced supercoiling for the function of the Gin enhancer in recombination but not for the activity of the gix sites.

\section{The resolvase synaptic complex as a recombination intermediate}

Our division of the resolvase reaction into two stages is operational. There may be many synaptic intermediates, but we define the synaptic complex as that which accumulates at $15^{\circ} \mathrm{C}$ and survives our analytical procedures; all reaction steps after the appearance of the synaptic complex are called postsynaptic. There are several lines of evidence showing that the operationally defined resolvase synaptic complex is an obligatory recombination intermediate. First, the synaptic complex can be directly converted into product (rapidly at $37^{\circ} \mathrm{C}$ and slowly at $15^{\circ} \mathrm{C}$ ) even after the addition of competitor DNA and dilution of the reaction sufficient to block resolvase binding to DNA (data not shown). Second, formation of the synaptic complex shares many of the requirements for recombination, notably two resolvase-bound res sites in a supercoiled molecule (Benjamin \& Cozzarelli, 1988; Parker \& Halford, 1991). Third, the synaptic complex trapped at $15^{\circ} \mathrm{C}$ contains three (-) plectonemic supercoils, as does the reaction intermediate at $37^{\circ} \mathrm{C}$ (Wasserman et al., 1985; Benjamin \& Cozzarelli, 1988, 1990; Dröge \& Cozzarelli, 1989; Stark et al., 1989; Stark \& Boocock, 1994). Fourth, the synaptic complex can be isolated without lowering reaction temperature to $15^{\circ} \mathrm{C}$. We found that the synaptic complex appeared at $37^{\circ} \mathrm{C}$, but then decayed quickly (Figure 2 and data not shown). High levels of synaptic complex were observed at $37^{\circ} \mathrm{C}$ when the superhelical density was -0.02 or less, or when the substrate was a nicked catenane (Figures 2 and 6 and data not shown). In the latter case, we showed that the topology of the synaptic complex was the same as that with supercoiled catenanes (Figures 8 and 9).

We are confident, therefore, that the synaptic complex we study is a true reaction intermediate. We do not know, however, whether all six subsites are held together in the synaptic complex (Figure 1(a)). In particular, the synapsis of subsite I might always be transient, because it leads immediately to recombination (Bednarz et al., 1990; Grindley, 1994; Rice \& Steitz, 1994a,b).

\section{Tn 3 resolvase and Gin have twofold requirements for supercoiling}

Tn3 resolvase synapsis requires either supercoiling or catenation. The presence of both increases synapsis. Although nicked circular DNA did not synapse (Figure 6), synapsis increased linearly with superhelical density to a plateau at -0.03 (Figure 2). Although unlinked rings did not synapse (Benjamin \& Cozzarelli, 1988), the efficient synapsis of nicked catenanes (Figure 6) demonstrated that catenation alone was sufficient for synapsis by resolvase. Therefore, synapsis requires the conformational roles of supercoiling.

Three lines of evidence conclusively establish a requirement for supercoiling in postsynaptic steps of the $\operatorname{Tn} 3$ resolvase reaction. First, recombination required higher superhelical density than did synapsis (Figure 2). Second, postsynaptic events required supercoiling outside the synaptic complex because relaxed synaptic complexes did not recombine (Figure 3). Finally, catenanes needed supercoiling for postsynaptic steps, but not for synapsis (Figures 5 and 6).

A similar result has been observed for recombination of hix sites by Hin, a system very similar to gix/Gin (Johnson \& Bruist, 1989; Lim \& Simon, 1992). The dependence on $\sigma$ of Hin reaction intermediates and products (Lim \& Simon, 1992) is similar to that of Tn 3 resolvase (Figure 2). For half maximal formation, the paired hix intermediate required a $\sigma$ of about -0.010 , whereas both the three-site synaptic complex and recombinants required a $\sigma$ of about -0.045 .

The caveat regarding our interpretation of the resolvase nicked catenane data is that the efficient synapsis and poor recombination we observe could arise from an aberrant association of res sites, rather than from a more stringent requirementfor supercoiling in postsynapsis. However, two lines of evidence indicate that the synaptic complexes on nicked catenanes are true intermediates. First, the number of nodes protected by the synaptic complexes from topoisomerase unlinking was identical for nicked and supercoiled catenane substrates (Figure 7). Second, under permissive conditions, Tn 3 resolvase recombination of fully-nicked catenanes produced only those recombinant species predicted by the standard mechanism (Figure 8). Therefore, it is highly 
likely that the synaptic complex formed under standard conditions was a trapped reaction intermediate.

Whereas the resolvase experiments offered insight into the synaptic and postsynaptic roles of supercoiling, Gin experiments pointed to different roles of supercoiling for the accessory and crossover sites. Because Gin recombination occurred when sis was on a nicked ring, the roles of supercoiling in the function of the accessory site are mimicked by catenation (Figure 10). However, no recombination occurred when a gix was on a nicked ring; therefore, a second role of supercoiling in the function of the crossover sites cannot be replaced by catenation. This is analogous to the Mu transposase recombinational enhancer that can act in trans when present on a linear fragment at a high concentration, although the reaction still requires supercoiled recombination sites (Surette \& Chaconas, 1992), suggesting that one major role of supercoiling (and catenation in this report) is to increase local site concentration.

Catenation can substitute for supercoiling in resolvase synapsis (Figure 6) and in the functioning of the Gin accessory site (Figure 10). Catenation alone cannot support $\mathrm{Tn} 3$ resolvase postsynaptic steps (Figure 5) or the functioning of the gix sites (Figure 10). If the FIS-bound sis were needed primarily for promoting synapsis or selecting the topologically correct synaptic complex for recombination (Haffter \& Bickle, 1988; Klippel et al., 1988a, 1993; Johnson \& Bruist, 1989; Kanaar et al., 1990; Heichman et al., 1991; Crisona et al., 1994), the results with Gin would imply that the contribution of supercoiling to synapsis can be replaced by catenation, as it is for resolvase.

Interestingly, supercoiling of only one crossover site was sufficient for resolvase recombination, but not for Gin inversion (Figures 5 and 10). It is possible that the $\operatorname{Tn} 3$ resolvase dimer bound to a supercoiled subsite I can transmit the benefit it gets from supercoiling to its partner dimer bound to a subsite I on a nicked ring. Perhaps due to the greater instability of the Gin synaptic complex (Kanaar, 1988) or the nature of the Gin interdimer contacts, a similar change cannot be transmitted by one Gin dimer to its partner.

The role of supercoiling in phage lambda Int recombination is rather different (Nash, 1990). Int is the archetype of a second family of site-specific recombinases that differs in amino acid sequence and mechanism from the resolvase/invertase family (Argos et al., 1986). For Int excisive recombination, neither recombination site need be on a supercoiled ring. For integrative recombination, one partner (attP) must be on a supercoiled molecule, whereas the other (attB) need not. From the elegant studies of the Nash and Landy groups, it seems clear that the essential role of supercoiling in integrative recombination is in promoting a specific nucleoprotein structure at attP (Richet et al., 1986; Thompson et al., 1987; Moitoso de Vargas et al., 1989).

\section{Comparison of catenated and supercoiled DNA}

Supercoiling has two distinct consequences for recombination: it limits the conformations of DNA and facilitates the unwinding of the double helix (Bates \& Maxwell, 1993; Vologodskii \& Cozzarelli, 1994). Catenanes are conformationally similar to supercoiled DNA, but cannot promote helix melting (Vologodskii \& Cozzarelli, 1993). Catenanes and superhelices share three conformational features that are potentially important for recombination. First, both supercoiling and catenation increase the local DNA site concentration by up to two orders of magnitude (Vologodskii et al., 1992; Vologodskii \& Cozzarelli, 1993). Second, the angle at which two DNA sites encounter each other has a narrow distribution centered at $60^{\circ}$ for supercoiled and catenated DNA rather than the broad distribution about $90^{\circ}$ for relaxed DNA (Vologodskii \& Cozzarelli, 1994; Vologodskii, personal communication). Third, the plectonemic interwinding of DNA in both forms energetically favors any process that interwinds sites or binds (-) nodes, as occurs in the resolvase or Gin synapsis (Benjamin \& Cozzarelli, 1988, 1990; Dröge \& Cozzarelli, 1989; Kanaar et al., 1989). Supercoiling favors conformations of DNA in which direct sites align "in parallel" with an odd number of interwindings in the synaptic complex (three for resolvase) and inverted sites align with an even number of interwindings (two for Gin; Wasserman et al., 1985; Kanaar et al., 1988; Moskowitz et al., 1991; Stark \& Boocock, 1995). Catenanes and knots promote specific recombination in the same way. If the global topology of a catenane or knot does not provide the geometry and alignment required in the synaptic complex, it will not be productively recombined (reviewed by Sumners et al., 1995; Stark \& Boocock, 1995).

The major qualitative difference between nicked catenanes and (-) supercoiled DNA involves twist. Twist of nicked catenanes is the same as that of nicked circles, whereas (-) supercoiling reduces twist (Richardson et al., 1988; Vologodskii \& Cozzarelli, 1993) and therefore favors any process that is accompanied by reduced twist. For example, the local unwinding or toroidal wrapping of DNA by a protein is favored by (-) supercoiling, but not by catenation.

Structural studies have elucidated the relationship between supercoiling and catenation. For comparison, it is convenient to define a catenation density, $\rho$, that is the cognate of $\sigma$ (Vologodskii \& Cozzarelli, 1993); $\rho$ is the number of catenane nodes normalized to the size of the rings. The three key conformational features of supercoiled DNA and catenanes (local site concentration, angle of site juxtaposition and plectonemic node density) have a dependence on $\rho$ similar to that on $\sigma$ (Vologodskii \& Cozzarelli, 1993). However, the catenanes used in this study have a $\rho$ value much less than the $\sigma$ of physiologically supercoiled DNA. The catenane 
products of the Int reaction have an average of 8.6 nodes, which corresponds to a $\rho$ value of -0.012 . In contrast, the supercoiled circular DNA has a $\sigma$ of -0.065 . Therefore, a failure of catenation to mimic supercoiling fully could be the result of this quantitative difference.

Because catenation can replace supercoiling for synapsis, the features of supercoiling required for synapsis must be conformational. This is well illustrated by a comparison of the dependence of synapsis on $\rho$ and $\sigma$. Synapsis of fully-nicked catenanes with an average $\rho$ of -0.012 was $60 \%$ that of supercoiled DNA with a $\sigma$ of -0.065 (Figure 6(a)). Synapsis of supercoiled DNA with a $\sigma$ of -0.015 was also $60 \%$ that of supercoiled DNA with a $\sigma$ of -0.065 (Figure 2). Therefore, $\rho$ is equivalent to $\sigma$ for synapsis.

\section{Roles of supercoiling for postsynapsis and gix sites}

The postsynaptic roles of supercoiling in Tn3 resolvase recombination are not as clearly defined as the synaptic ones. Our result, showing that the supercoiling requirement is more stringent for postsynapsis, can be interpreted in two ways. The same conformational features of supercoiling that are critical for synapsis might be required at higher levels for postsynaptic steps. According to this interpretation, the nicked catenanes did not recombine because their $\rho$ is too low. Alternatively, there are additional roles of supercoiling needed for postsynapsis that are not provided by fully-nicked catenanes regardless of their complexity. According to this view, postsynapsis requires a torsional feature of supercoiling that can never be provided by catenation.

Unfortunately, catenanes with the same number of nodes as physiologically supercoiled substrates are not available. However, one observation suggests that different roles of supercoiling, not just different amounts, are required postsynaptically. If catenane recombination were limited only by the values of $\rho$, then an 18-noded catenane with $\rho=-0.026$ should recombine to a level 15 to $20 \%$ that of supercoiled DNA (Figure 2). Yet, even after 17 hours incubation, Tn3 resolvase failed to recombine an 18 -noded catenane $(<0.1 \%$, data not shown). Although $\rho$ is a good predictor of synapsis, it is a poor predictor of postsynapsis. Therefore, factors other than local concentration, angle of site incidence, and plectonemic node density are important postsynaptically.

Unidirectional rotation of res sites during exchange could be driven by the conformation of (-) supercoiled DNA. Other than this, it seems that the dependence of postsynapsis on supercoiling must result from a local torsional effect rather than a global conformational effect. The only reasonable candidate is unwinding of the duplex. A supercoiling-independent Gin mutant unwound a region near the scissile bonds of gix on a linear DNA, whereas the wild-type Gin did not (Klippel et al.,
1993). This clearly implicates a role of supercoiling for Gin in melting the DNA at the crossover site, a role which may also be important postsynaptically for resolvase. Because catenation cannot promote unwinding and we have shown that catenation supports synapsis, any unwinding must be postsynaptic. The rate-limiting step for resolvase is after synapsis (Parker \& Halford, 1991), and may be a supercoiling-dependent rearrangement of the synaptic complex involving unwinding at the A + T-rich subsite I (Hatfull et al., 1987; Klippel et al., 1988b; Castell \& Halford, 1989; Mazzarelli et al., 1993; Stark \& Boocock, 1995). By necessity, the two base-pairs between the scissile bonds must be unpaired before the halfsites are exchanged, and the melting may occur even before cleavage. In the crystal structures of resolvase, the nucleophiles are quite far apart (Rice \& Steitz, 1994a,b; Yang \& Steitz, 1995). Supercoiling-dependent double helix melting could position the scissile bonds near the nucleophiles or facilitate a catalytic activation of the proteins (Klippel et al., 1993; Grindley, 1994). Supercoiling might also promote strand exchange (not just dictate its direction) by coupling it to a release of tension established in the synaptic complex. Structural studies of resolvase have led to the suggestion that the crossover site may be "cocked" in the activated complex so that cleavage results in the immediate release of tension by rotation (Rice \& Steitz, 1994a).

Despite the stringent postsynaptic need for supercoiling, it can be bypassed partly or completely in a number of ways. We discovered that the postsynaptic roles of supercoiling can be partially replaced by catenation for $\gamma \delta$ but not Tn 3 resolvase (Figure 9). It is evident from past work that knotting also suffices for $\gamma \delta$ resolvase (Dröge, 1992). Tn 3 resolvase can recombine nicked singly-linked catenane substrates that contain a very small ring and a large ring, because the release of strain in the small ring drives the reaction (Stark et al., 1989). A variety of altered reaction conditions have been shown to permit supercoiling-independent recombination by resolvase and $\mathrm{Mu}$ transposase (Dröge \& Cozzarelli, 1989; Stark et al.,1989; Baker \& Mizuuchi, 1992; see also Figure 8).

Our work has illustrated that relatively small differences in proteins that share a reaction mechanism can nonetheless result in very different dependencies on supercoiling. For example, one res can be on a nicked ring, but one gix cannot (Figures 5 and 10). Also, $\gamma \delta$ resolvase can recombine nicked catenanes, but Tn 3 resolvase cannot (Figures 5 and 9). Halford and colleagues have reported that $\operatorname{Tn} 21$ resolvase recombination is even more sensitive to supercoiling than $\mathrm{Tn} 3$ resolvase (Oram et al., 1994). More extreme examples are the supercoiling-independent Gin, Cin, Hin, and resolvase mutants that contain a single amino acid change (Haffter \& Bickle, 1988; Klippel et al., 1988a; R. C. Johnson, personal communication; M. R. Boocock, X. Zhu, N. D. F. Grindley \& W. Yang, personal communication). Such large differences 
between enzymes of similar amino acid sequence, mechanism and function suggest a teleological explanation of supercoiling dependence (Klippel et al., 1993; Crisona et al., 1994; Stark \& Boocock, 1995). The mechanisms of recombinases may be constructed to depend on supercoiling as a regulatory signal and selectivity determinant, rather than as an obligatory contributor to chemistry or thermodynamics. On this hypothesis, the reaction could easily be independent of supercoiling, but only by sacrificing selectivity and regulation. This illustrates why the multiple roles of supercoiling in site-specific recombination can be bypassed in a number of ways.

\section{Materials and Methods}

\section{Proteins}

Both resolvases were purified as described (Hatfull et al., 1989) with minor modifications including the use of a MonoS column (Pharmacia HR5/5) instead of DE52. The resolvase eluted near the midpoint of a 20-columnvolume linear gradient from 0 to $300 \mathrm{mM} \mathrm{NaCl}$.

Bacteriophage $\lambda$ Int (Nash, 1983), E. coli IHF (Nash et al., 1987) and FIS (Koch \& Kahmann, 1986), bacteriophage Mu Gin (Mertens et al., 1986; Kanaar et al., 1988), T2 topoisomerase II (Kreuzer \& Jongeneel, 1983), and wheat germ topoisomerase I (Dynan et al., 1981) were purified as described. Bacteriophage fd gpII (Meyer \& Geider, 1979) was a generous gift of B. Alberts, University of California at San Francisco. Restriction enzymes and bovine pancreatic DNase I were from commercial sources.

\section{Resolvase and Gin substrate preparation}

We designate our plasmid substrates by the recombination sites they contain. Thus, pResInt contains two directly repeated $\gamma \delta$ resolvase sites res separated by the Int sites att $\mathrm{B}$ and att $\mathrm{P}$, also directly repeated (Figure 11(a)). It was constructed by insertion of the $393 \mathrm{bp}$ BamHI fragment from pJMC110 that contains the M13 ori (Cleary \& Ray, 1980) into the BglII site of pAB7.0d, which has the recombination sites (Benjamin \& Cozzarelli, 1990). After recombination (Figure 4), digestion with NdeI and $\mathrm{Bam} \mathrm{HI}$ yields three fragments, one of which is unique to each of the four relevant DNAs: substrate $(S), 4.5 \mathrm{~kb}$; resolvase product $(\mathrm{R}), 4.35 \mathrm{~kb}$; Int product (I), $3.85 \mathrm{~kb}$; and product of Int then resolvase (IR), $3.7 \mathrm{~kb}$. Reaction with phage fd gpII selectively nicks the M13 ori (Meyer \& Geider, 1979). The laboratory name for pResInt is pRKS6.

The Gin substrates, pGinInt(Ns) and pGinInt(Ng), both have gix sites in inverted orientation interspersed with directly repeated att $\mathrm{P}$ and $a t t \mathrm{~B}$, and the $\mathrm{M} 13$ ori and sis (Figure 11(b) and (c)). The parenthetical designation indicates that after reaction with Int and gpII, the nicked (N) ring contains the sis (s) or a gix (g) site. The hemi-nicked catenanes produced from these plasmids are called hemi-nicked (sis) or hemi-nicked (gix). pGinInt(Ns) was constructed by insertion of the BamHI fragment from pJMC110 (Cleary \& Ray, 1980) that contains the M13 ori into the BamHI site of pGP522 (Kanaar et al., 1989); this plasmid is also known as pRKS2. Int catenanes from pGinInt(Ns) have sis and the M13 ori on one ring and both gix sites on the other ring. Digestion with EcoRI and BamHI discriminates among the
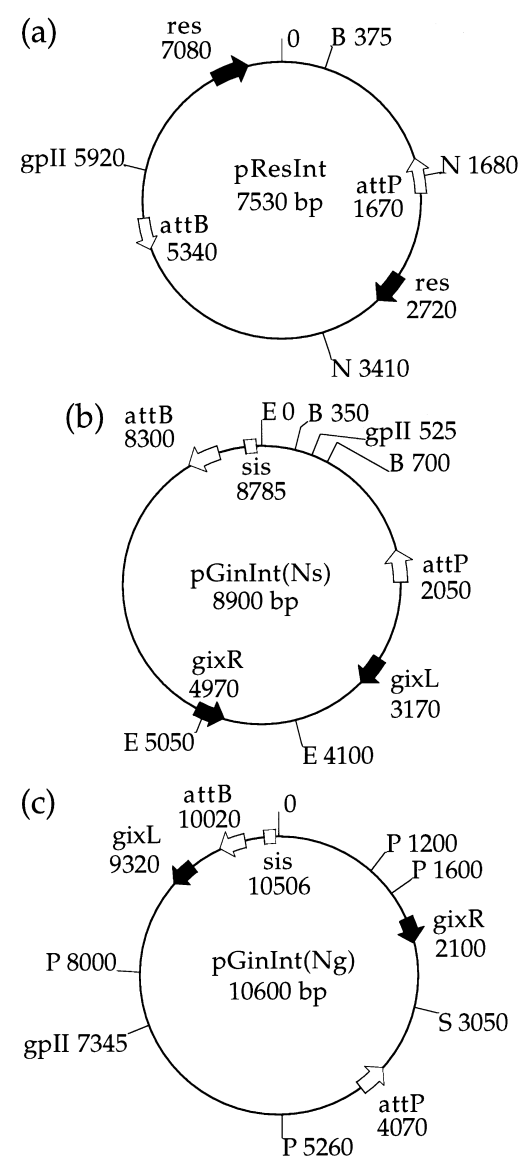

Figure 11. Plasmid maps of the resolvase and Gin substrates. The recombination sites for resolvase (res), Gin (gix), and Int (att) are indicated. The Gin enhancer (sis) and the site of gpII nicking are also shown. The sites are not drawn to scale. Relevant restriction enzyme sites are designated by abbreviations: B, BamHI; E, EcoRI; N, NdeI; P, PvuII; S, SalI. Numbers reflect the approximate distance in base-pairs from position zero to the middle of the sites. (a) pResInt. (b) pGinInt(Ns). (c) pGinInt(Ng). The Gin substrates can be converted into hemi-nicked (sis) and hemi-nicked (gix), respectively.

recombination products. pGinInt $(\mathrm{Ng})$ was constructed by insertion of the $1.3 \mathrm{~kb} P v u \mathrm{I}$ fragment of pUC118, containing the M13 ori, into a PvuI site of pGP541 (Kanaar et al., 1989); this plasmid is also known as pRKS3. Int catenanes from $p$ GinInt $(\mathrm{Ng})$ have sis and a gix on one ring and a gix and the M13 ori on the other ring. Cleavage with SalI and PvuII produces fragments diagnostic of recombination.

Populations of pResInt with different average $\sigma$ values were produced by reaction with wheat germ topoisomerase I in resolvase reaction buffer supplemented with ethidium bromide, as described previously (Benjamin et al., 1985).

Int reactions to create supercoiled catenanes were as described (Benjamin \& Cozzarelli, 1990), except less protein was added $(3.25 \mu \mathrm{g} / \mathrm{ml}$ Int and $2.5 \mu \mathrm{g} / \mathrm{ml}$ integration host factor (IHF)). After reaction with Int, a fraction of the DNA was nicked with DNase I to generate fully-nicked catenanes (Crisona et al., 1994). Phage fd gpII reactions to produce hemi-nicked catenanes contained $57 \mu \mathrm{g} / \mathrm{ml}$ DNA, $20 \mathrm{mM}$ Tris- $\mathrm{HCl}$ (pH 8.0), $50 \mathrm{mM} \mathrm{NaCl}$, $2 \mathrm{mM} \mathrm{MgCl}_{2}, 2 \mathrm{mM}$ 2-mercaptoethanol, 5\% glycerol, $0.1 \mathrm{mg} / \mathrm{ml} \mathrm{BSA}$, and $5.7 \mu \mathrm{g} / \mathrm{ml}$ gpII. Incubation was for 
30 minutes at $30^{\circ} \mathrm{C}$. All large-scale preparative reactions were stopped by adding EDTA to $5 \mathrm{mM}$ and SDS to $0.5 \%$, inactivating with heat, or both. Before reaction with resolvase or Gin, the DNA was extracted and precipitated using standard techniques, and then assayed for recovery and efficiency of preparative reactions. Int reactions were usually $70 \%$ complete, as determined by electrophoretic separation of diagnostic restriction enzyme fragments. Supercoiling or nicking of each ring in a catenane was analyzed by cutting one ring with a restriction enzyme, followed by electrophoretic separation to determine whether the intact circle was supercoiled or nicked. Nicking was generally $99 \%$ complete and supercoiled preparations were less than $10 \%$ nicked.

Purified catenane isomers were isolated from reactions with Int followed by DNase I nicking. The catenanes were separated by high-resolution gel electrophoresis and recovered from gel slices (Crisona et al., 1994).

\section{Resolvase and Gin reactions}

Standard resolvase reactions contained $20 \mathrm{mM}$ Hepes (pH 7.5), $10 \mathrm{mM} \mathrm{MgCl}$, $175 \mathrm{mM} \mathrm{NaCl}, 10$ to $30 \mu \mathrm{g} / \mathrm{ml}$ DNA, and a 40-fold molar ratio of resolvase monomer to DNA. Recombination was usually measured after 60 minutes at $37^{\circ} \mathrm{C}$ and synapsis after one minute at $15^{\circ} \mathrm{C}$. Recombination and synapsis were assayed as described, with minor variations (Krasnow \& Cozzarelli, 1983; Benjamin \& Cozzarelli, 1988). Recombination reactions were stopped by heat killing or phenol/chloroform (1:1, $\mathrm{v} / \mathrm{v}$ ) extraction and synapsis reactions by crosslinking resolvase with $0.2 \%$ glutaraldehyde. After ethanol precipitation or gel filtration (CL-4B, Pharmacia), respectively, the DNA was digested with restriction enzymes at $37^{\circ} \mathrm{C}$. Digestion of both loops outside the synaptic complex produced a $\chi$-shaped structure that was detected by its characteristic gel mobility (Benjamin \& Cozzarelli, 1988).

To remove supercoiling from the DNA outside of the Tn 3 resolvase synaptic complex, synapsis reactions were treated with excess wheat germ topoisomerase I for five minutes at $15^{\circ} \mathrm{C}$. A portion of the reaction was analyzed for synapsis and relaxation, and the remainder was shifted to $37^{\circ} \mathrm{C}$ to facilitate recombination. Synapsis and relaxation were measured by glutaraldehyde crosslinking and restriction enzyme cleavage of one DNA loop outside the synaptic complex to produce an $\alpha$ structure (Benjamin \& Cozzarelli, 1988) whose electrophoretic mobility depends on whether the intact loop is supercoiled or relaxed.

To determine how many catenane crossings the synaptic complex protected from topoisomerase unlinking, the standard synapsis protocol was modified such that the gel filtration step was also used for buffer exchange. After adjustment to $10 \mathrm{mM}$ Tris- $\mathrm{HCl}$ (pH 7.5), $50 \mathrm{mM} \mathrm{KCl}, 50 \mathrm{mM} \mathrm{NaCl}, 5 \mathrm{mM} \mathrm{MgCl}, 0.1 \mathrm{mM}$ EDTA, $1 \mathrm{mM}$ ATP, 2.5\% (v/v) glycerol, $50 \mu \mathrm{g} / \mathrm{ml} \mathrm{BSA}$, and $11 \mu \mathrm{g} / \mathrm{ml}$ DNA, the eluent was treated with 0.9 or $1.8 \mu \mathrm{g} / \mathrm{ml} \mathrm{T} 2$ topoisomerase for 30 minutes at $30^{\circ} \mathrm{C}$. Half of each topoisomerase reaction was assayed for synapsis by the mobility shift assay. The other half was extracted, precipitated, nicked with DNase I, and run on a high-resolution gel.

Under permissive resolvase recombination conditions, supercoiling was not required for $\operatorname{Tn} 3$ resolvase recombination of nicked catenanes or knots (Dröge \& Cozzarelli, 1989; see also Figure 8). Reactions contained EDTA and spermidine and lacked $\mathrm{Mg}^{2+}$ (Dröge \& Cozzarelli, 1989). The reactions shown were for 17 hours at $37^{\circ} \mathrm{C}$, but the reaction was $20 \%$ complete by 30 minutes. Gin reactions contained $20 \mathrm{mM}$ Tris-Cl (pH 7.5), $130 \mathrm{mM} \mathrm{NaCl}, 10 \mathrm{mM} \mathrm{MgCl}_{2}, 5 \%$ glycerol, $15 \mu \mathrm{g} / \mathrm{ml}$ DNA, $1.8 \mu \mathrm{g} / \mathrm{ml} \mathrm{Gin}$, and $0.97 \mu \mathrm{g} / \mathrm{ml}$ FIS. Incubation at $37^{\circ} \mathrm{C}$ for 60 minutes was followed by heat inactivation, restriction enzyme digestion, and electrophoresis.

\section{Gel electrophoresis, Southern blotting, and DNA quantification}

Products of restriction enzyme digestion were separated by electrophoresis through $0.8 \%(\mathrm{w} / \mathrm{v})$ agarose Tris-borate, EDTA (TBE) gels. For analysis of synapsis, the wells were dry-loaded and run for 15 minutes at $1.3 \mathrm{~V} / \mathrm{cm}$, then covered with buffer and continued at $3.3 \mathrm{~V} / \mathrm{cm}$. High resolution electrophoretic separation of nicked catenanes and knots was through a $0.8 \%$ or $1 \%$ agarose gel in a Tris-acetate, EDTA (TAE) buffer containing SDS (Sundin \& Varshavsky, 1981). All gels were stained with ethidium bromide and photographed on a UV transilluminator. The intensities of DNA fragments imaged in the negatives were quantitated with a laser densitometer (LKB).

Southern blotting using MSI Magna membrane was followed by UV crosslinking and hybridization at $68^{\circ} \mathrm{C}$ for at least 12 hours with a ${ }^{32} \mathrm{P}$-radiolabeled, nick-translated substrate probe. Blots were quantitated with a Molecular Dynamics phosphorimager.

The amount of DNA synapsed or recombined was calculated as follows. First, the intensity of each gel band was normalized to the length of DNA in the band to give relative molar amounts of the fragments diagnostic of reacted $(R)$ or parental $(P)$ species. Then, for each lane, the fraction of reacted DNA was calculated using the formula $\mathrm{R} /(\mathrm{R}+\mathrm{P})$. In most figures, the percentage of DNA reacted is presented. In Figure 2, to facilitate comparison of synapsis with recombination, the percentage of DNA reacted was normalized to the highest value obtained for the assay in that experiment.

\section{Acknowledgments}

We thank Kathy Collins, Alexander Vologodskii, and members of the lab for helpful comments on the manuscript. This work was supported by NIH grants GM31655 and GM31657, and NIEHS grant ESO1896 to NRC. K.R.B. was a Graduate Research Fellow of the National Science Foundation. A.P.A. was supported by Public Health Service training grants GM08295 and GM07232. R.K. was a fellow of The Jane Coffin Childs Memorial Fund for Medical Research and is currently a fellow of the Royal Netherlands Academy of Arts and Sciences.

\section{References}

Adams, D. E., Shekhtman, E. M., Zechiedrich, E. L., Schmid, M. B. \& Cozzarelli, N. R. C. (1992). The role of topoisomerase IV in partitioning bacterial replicons and the structure of catenated intermediates in DNA replication. Cell, 71, 277-288.

Argos, P., Landy, A., Abremski, K., Egan, J. E., Haggard-Ljungquist, E., Hoess, R. H., Kahn, M. L., Kalionis, B., Narayana, S. V. L., Pierson, L. S., Sternberg, N. \& Leong, J. M. (1986). The integrase family of site-specific recombinases: regional similarities aind global diversity. EMBO J. 5, 433-440. 
Baker, T. A. \& Mizuuchi, K. (1992). DNA-promoted assembly of the active tetramer of the $\mathrm{Mu}$ transposase. Genes Dev. 6, 2221-2232.

Bates, A. D. \& Maxwell, A. (1993). DNA Topology, Oxford University Press, New York, USA.

Bednarz, A. L., Boocock, M. R. \& Sherratt, D. J. (1990). Determinants of correct res site alignmeint in site-specific recombination by $\operatorname{Tn} 3$ resolvase. Genes Dev. 4, 2366-2375.

Benjamin, H. W. \& Cozzarelli, N. R. (1988). Isolation and characterization of the $\mathrm{Tn} 3$ resolvase synaptic intermediate. EMBO J. 7, 1897-1905.

Benjamin, H. W. \& Cozzarelli, N. R. (1990). Geometric arrangements of Tn3 resolvase sites. J. Biol. Chem. 265, 6441-6447.

Benjamiin, H. W., Matzuk, M. M., Krasnow, M. A. \& Cozzarelli, N. R. (1985). Recombination site selection by $\operatorname{Tn} 3$ resolvase: topological tests of a tracking mechanism. Cell, 40, 147-158.

Bliska, J. B., Benjamin, H. W. \& Cozzarelli, N. R. (1991). Mechanism of $\operatorname{Tn} 3$ resolvase recombination in vivo. J. Biol. Chem. 266, 2041-2047.

Castell, S. E. \& Halford, S. E. (1989). DNA supercoiling determines the activation energy barrier for sitespecific recombination by Tn21 resolvase. Nucl. Acids Res. 17, 7045-7058.

Cleary, J. M. \& Ray, D. S. (1980). Replication of the plasmid pBR322 under control of a cloned replication origin from single-stranded DNA phage M13. Proc. Natl Acad. Sci. USA, 77, 4638-4642.

Cozzarelli, N. R. \& Wang, J. C. (eds; 1990). DNA Topology and its Biological Effects, Cold Spring Harbor Laboratory Press, Cold Spring Harbor, New York, USA.

Craigie, R. \& Mizuuchi, K. (1986). Role of DNA topology in $\mathrm{Mu}$ transposition: mechanism of sensing the relative orientation of two DNA segments. Cell, 45, 793-800.

Crisona, N. J., Kanaar, R., Gonzalez, T. N., Zechiedrich, E. L., Klippel, A. \& Cozzarelli, N. R. (1994). Processive recombination by wild-type Gin and an enhancer-independent mutant: insight into the mechanisms of recombination selectivity and strand exchange. J. Mol. Biol. 243, 437-457.

Dröge, P. (1992). Recombination of nicked DNA knots by $\gamma \delta$ resolvase suggests a variant model for the mechanism of strand exchange. Nucl. Acids Res. 20, 6159-6166.

Dröge, P. \& Cozzarelli, N. R. (1989). Recombination of knotted substrates by Tn 3 resolvase. Proc. Natl Acad. Sci. USA, 86, 6062-6066.

Dynan, W. S., Jendrisak, J. J., Hager, D. A. \& Burgess, R. R. (1981). Purification and characterization of wheat germ DNA topoisomerase I (nicking-closing enzyme). J. Biol. Chem. 256, 5860-5865.

Feng, J.-A., Dickerson, R. E. \& Johnson, R. C. (1994). Proteins that promote DNA inversion and deletion. Curr. Opin. Struct. Biol. 4, 60-66.

Glasgow, A. C., Hughes, K. T. \& Simon, M. I. (1989). Bacterial DNA inversion systems. In Mobile DNA (Berg, D. E. \& Howe, M. M., eds), pp. 637-659, American Society for Microbiology, Washington, DC.

Grindley, N. D. F. (1994). Resolvase-mediated site-specific recombination. In Nucleic Acids and Molecular Biology (Eckstein, F. \& Lilley, D. M. J., eds), vol. 8, pp. 236-267, Springer-Verlag, New York, USA.

Haffter, P. \& Bickle, T. A. (1988). Enhancer-independent mutants of the Cin recombinase have a relaxed topological specificity. EMBO J. 7, 3991-3996.

Hatfull, G. F. \& Grindley, N. D. F. (1986). Analysis of $\gamma \delta$ resolvase mutants in vitro: evidence for an interaction between serine 10 of resolvase and site I of res. Proc. Natl Acad. Sci. USA, 83, 5429-5433.

Hatfull, G. F. \& Grindley, N. D. F. (1988). Resolvases and DNA-invertases: a family of enzymes active in site-specific recombination. In Genetic Recombination (Kucherlapati, R. \& Smith, G. R., eds), pp. 357-396, American Society for Microbiology, Washington, DC.

Hatfull, G. F., Noble, S. M. \& Grindley, N. D. F. (1987). The $\gamma \delta$ resolvase induces an unusual DNA structure at the recombination crossover point. Cell, 9, 103-110.

Hatfull, G. F., Sanderson, M. R., Freemont, P. S., Raccuia, P. R., Grindley, N. D. F. \& Steitz, T. A. (1989). Preparation of heavy-atom derivatives using site-directed mutagenesis: introduction of cysteine residues into $\gamma \delta$ resolvase. J. Mol. Biol. 208, 661-667.

Heichman, K. A. \& Johnson, R. C. (1990). The Hin invertasome: protein-mediated joining of distant recombination sites at the enhancer. Science, 249 511-517.

Heichmain, K. A., Moskowitz, I. P. G. \& Johnson, R. C. (1991). Configuration of DNA strands and mechanism of strand exchange in the Hin invertasome as revealed by analysis of recombinant knots. Genes Dev. 5, 1622-1634.

Johnson, R. C. (1991). Mechanism of site-specific DNA inversion in bacteria. Curr. Opin. Genet. Dev. 1, 404-407.

Johnson, R. C. \& Bruist, M. F. (1989). Intermediates in Hin-mediated DNA inversion: a role for Fis and the recombinational enhancer in the strand exchange reaction. EMBO J. 8, 1581-1590.

Johnson, R. C. \& Simon, M. I. (1985). Hin-mediated site-specific recombination requires two $26 \mathrm{bp}$ recombination sites and a $60 \mathrm{bp}$ recombinational enhancer. Cell, 41, 781-791.

Kanaar, R. (1988). Site-specific DNA inversion in the genome of bacteriophage $\mathrm{Mu}$ : a topological analysis. Ph.D. thesis, University of Leiden.

Kanaar, R. \& Cozzarelli, N. R. (1992). Roles of supercoiled DNA structure in DNA transactions. Curr. Opin. Struct. Biol. 2, 369-379.

Kanaar, R., vain de Putte, P. \& Cozzarelli, N. R. (1988). Gin-mediated DNA inversion: product structure and the mechanism of strand exchange. Proc. Natl Acad. Sci. USA, 85, 752-756.

Kanaar, R., van de Putte, P. \& Cozzarelli, N. R. (1989). Gin-mediated recombination of catenated and knotted substrates: implications for the mechanism of interaction between cis-acting sites. Cell, 58, 147-159.

Kanaar, R., Klippel, A., Shekhtman, E., Dungan, J., Kahmann, R. \& Cozzarelli, N. R. (1990). Processive recombination by the phage $\mathrm{Mu}$ Gin system: implications for the mechanisms of DNA strand exchange, DNA site alignment, and enhancer action. Cell, 62, 353-366.

Klippel, A., Cloppenborg, K. \& Kahmann, R. (1988a). Isolation and characterization of unusual Gin mutants. EMBO J. 7, 3983-3989.

Klippel, A., Mertens, G., Patschinsky, T. \& Kahmann, R. (1988b). The DNA invertase Gin of phage Mu: formation of a covalent complex with DNA via a phosphoserine at amino acid position 9. EMBO J. 7, 1229-1237.

Klippel, A., Kanaar, R., Kahmann, R. \& Cozzarelli, N. R. (1993). Analysis of strand exchange and DNA binding of enhancer-independent Gin recombinase mutants. EMBO J. 12, 1047-1057.

Koch, C. \& Kahmann, R. (1986). Purification and properties of the Escherichia coli host factor required 
for inversion of the $\mathrm{G}$ segment in bacteriophage $\mathrm{Mu}$. J. Biol. Chem. 261, 15673-15678.

Krasnow, M. A. \& Cozzarelli, N. R. (1983). Site-specific relaxation and recombination by the $\operatorname{Tn} 3$ resolvase: recognition of the DNA path between oriented res sites. Cell, 32, 1313-1324.

Kreuzer, K. N. \& Jongeneel, C. V. (1983). Escherichia coli phage T4 topoisomerase. Methods Enzymol. 100, 144-160.

Lim, H. M. \& Simon, M. I. (1992). The role of negative supercoiling in Hin-mediated site-specific recombination. J. Biol. Chem. 267, 11176-11182.

Mazzarelli, J. M., Ermacora, M. R., Fox, R. O. \& Grindley, N. D. F. (1993). Mapping interactions between the catalytic domain of resolvase and its DNA substrate using cysteine-coupled EDTA-iron. Biochemisty, 32, 2979-2986.

Mertens, G., Hoffman, A., Blocker, H., Frank, R. \& Kahmann, R. (1984). Gin-mediated site-specific recombination in bacteriophage $\mathrm{Mu}$ DNA: overproduction of the protein and inversion in vitro. EMBO J. 3, 2415-2421.

Mertens, G., Fuss, H. \& Kahmann, R. (1986). Purification and properties of the DNA invertase Gin encoded by bacteriophage Mu. J. Biol. Chem. 261, 15668-15672.

Meyer, T. F. \& Geider, K. (1979). Bacteriophage fd gene II-protein. II. Specific cleavage and relaxation of supercoiled RF from filamentous phages. J. Biol. Chem. 254, 12642-12646.

Moitoso de Vargas, L., Kim, S. \& Landy, A. (1989). DNA looping generated by DNA bending protein IHF and the two domains of lambda integrase. Science, 244, 1457-1461.

Moskowitz, I. P. G., Heichman, K. A. \& Johnson, R. C. (1991). Alignment of recombination sites in Hin-mediated site-specific DNA recombination. Genes Dev. 5, 1635-1645.

Nash, H. A. (1983). Purification and properties of the bacteriophage lambda Int protein. Methods Enzymol. 100, 210-216.

Nash, H. A. (1990). Bending and supercoiling of DNA at the attachment site of bacteriophage $\lambda$. Trends Biochem. Sci. 15, 222-227.

Nash, H. A., Robertson, C. A., Flamm, E., Weisberg, R. A. \& Miller, H. I. (1987). Overproduction of Escherichia coli integration host factor, a protein with nonidentical subunits. J. Bacteriol. 169, 4124-4127.

Oram, M., Shipstone, E. \& Halford, S. E. (1994). Synapsis by Tn 3 resolvase: speed and dependence on DNA supercoiling. Biochem. Soc. Trans. 22, 303S.

Parker, C. N. \& Halford, S. E. (1991). Dynamics of long-range interactions on DNA: the speed of synapsis during site-specific recombination by resolvase. Cell, 66, 781-791.

Plasterk, R. H. A., Kanaar, R. \& van de Putte, P. (1984). A genetic switch in vitro: DNA inversion by Gin protein of phage Mu. Proc. Natl Acad. Sci. USA, 81, 2689-2692.

Reed, R. R. (1981). Transposon-mediated site-specific recombination: a defined in vitro system. Cell, 25, 713-719.

Rice, P. A. \& Steitz, T. A. (1994a). Model for a DNA mediated synaptic complex suggested by crystal packing of $\gamma \delta$ resolvase subunits. EMBO J. 13, 1514-1524.

Rice, P. A. \& Steitz, T. A. (1994b). Refinement of $\gamma \delta$ resolvase reveals a strikingly flexible molecule. Structure, 2, 371-384.

Richardson, S. M., Boles, T. C. \& Cozzarelli, N. R. (1988). The helical repeat of underwound DNA in solution. Nucl. Acids Res. 16, 6607-6616.

Richet, E., Abcarian, P. \& Nash, H. A. (1986). The interaction of recombination proteins with supercoiled DNA: defining the role of supercoiling in lambda integrative recombination. Cell, 46, 1011-1021.

Sherratt, D. (1989). Tn3 and related transposable elements: site-specific recombination and transposition. In Mobile DNA (Berg, D. E. \& Howe, M. M., eds), pp. 163-184, American Society for Microbiology, Washington, DC.

Stark, W. M. \& Boocock, M. R. (1994). The linkage change of a knotting reaction catalyzed by Tn3 resolvase. J. Mol. Biol. 239, 25-36.

Stark, W. M. \& Boocock, M. R. (1995). Topological selectivity in site-specific recombination. In Mobile Genetic Elements (Sherratt, D. J., ed.), pp. 101-129, IRL Press at Oxford University Press, Oxford, UK.

Stark, W. M., Sherratt, D. J. \& Boocock, M. R. (1989). Site-specific recombination by Tn 3 resolvase: topological changes in the forward and reverse reactions. Cell, 58, 779-790.

Stark, W. M., Grindley, N. D. F., Hatfull, G. F. \& Boocock, M. R. (1991). Resolvase-catalysed reactions between res sites differing in the central dinucleotide of subsite I. EMBO J. 10, 3541-3548.

Sumners, D. W., Ernst, C., Spengler, S. J. \& Cozzarelli, N. R. (1995). Analysis of the mechanism of DNA recombination using tangles. Q. Rev. Biophys. 28, 253-313.

Sundin, O. \& Varshavsky, A. (1981). Arrest of segregation leads to accumulation of highly intertwined catenated dimers: dissection of final stages of SV40 DNA replication. Cell, 25, 659-669.

Surette, M. G. \& Chaconas, G. (1992). The Mu transpositional enhancer can function in trans. Requirement of the enhancer for synapsis but not strand cleavage. Cell, 68, 1101-1108.

Thompson, J. F., Moitoso de Vargas, L., Skinner, S. E. \& Landy, A. (1987). Protein-protein interactions in a higher-order structure direct lambda site-specific recombination. J. Mol. Biol. 195, 481-493.

Vologodskii, A. V. \& Cozzarelli, N. R. (1993). Monte Carlo analysis of the conformation of DNA catenanes. J. Mol. Biol. 232, 1130-1140.

Vologodskii, A. V. \& Cozzarelli, N. R. (1994). Conformational and thermodynamic properties of supercoiled DNA. Ann. Rev. Biophys. Biomol. Struct. 23, 609-643.

Vologodskii, A. V., Levene, S. D., Klenin, K. V., Frank-Kamenetskii, M. D. \& Cozzarelli, N. R. (1992). Conformational and thermodynamic properties of supercoiled DNA. J. Mol. Biol. 227, 1224-1243.

Wasserman, S. A. \& Cozzarelli, N. R. (1986). Biochemical topology: applications to DNA recombination and replication. Science, 232, 951-960.

Wasserman, S. A., Dungan, J. M. \& Cozzarelli, N. R. (1985). Discovery of a predicted DNA knot substantiates a model for site-specific recombination. Science, 229, 171-174.

Yang, W. \& Steitz, T. A. (1995). Crystal structure of the site-specific recombinase $\gamma \delta$ resolvase complexed with a 34 bp cleavage site. Cell, 82, 193-207. 